\title{
Regularity of weak solutions to the Navier- Stokes equations in exterior domains
}

\author{
Reinhard Farwig and Christian Komo
}

\begin{abstract}
Let $u$ be a weak solution of the Navier-Stokes equations in an exterior domain $\Omega \subset \mathbb{R}^{3}$ and a time interval $[0, T[, 0<T \leq \infty$, with initial value $u_{0}$, external force $f=\operatorname{div} F$, and satisfying the strong energy inequality. It is well known that global regularity for $u$ is an unsolved problem unless we state additional conditions on the data $u_{0}$ and $f$ or on the solution $u$ itself such as Serrin's condition $\|u\|_{L^{s}\left(0, T ; L^{q}(\Omega)\right)}<\infty$ with $2<s<\infty, \frac{2}{s}+\frac{3}{q}=1$. In this paper, we generalize results on local in time regularity for bounded domains, see Farwig et al. (Indiana Univ Math J 56:2111-2131, 2007; J Math Fluid Mech 11:1-14, 2008; Banach Center Publ 81:175-184, 2008), to exterior domains. If e.g. $u$ fulfills Serrin's condition in a left-side neighborhood of $t$ or if the norm $\|u\|_{L^{s^{\prime}}\left(t-\delta, t ; L^{q}(\Omega)\right)}$ converges to 0 sufficiently fast as $\delta \rightarrow 0+$, where $\frac{2}{s^{\prime}}+\frac{3}{q}>1$, then $u$ is regular at $t$. The same conclusion holds when the kinetic energy $\frac{1}{2}\|u(t)\|_{2}^{2}$ is locally Hölder continuous with exponent $\alpha>\frac{1}{2}$.
\end{abstract}

Mathematics Subject Classification (2000). Primary 76D05; Secondary 35Q30, 35B65.

Keywords. Instationary Navier-Stokes equations, Very weak solutions, Weak solutions, Serrin's class, Local in time regularity, Exterior domain.

\section{Introduction and main results}

In this paper, $\Omega \subset \mathbb{R}^{3}$ is an exterior domain, i.e. an open, connected subset having a nonempty, compact complement in $\mathbb{R}^{3}$, with smooth boundary $\partial \Omega \in C^{2,1}$, and $[0, T[, 0<T \leq \infty$, denotes the time interval. In $[0, T[\times \Omega$ we consider the instationary Navier-Stokes equations

$$
\begin{array}{rlrl}
u_{t}-\nu \Delta u+u \cdot \nabla u+\nabla p & =f & & \text { in }] 0, T[\times \Omega \\
\operatorname{div} u=0 & & \text { in }] 0, T[\times \Omega \\
u & =0 & & \text { on }] 0, T[\times \partial \Omega \\
u & =u_{0} & & \text { at } t=0
\end{array}
$$


with constant viscosity $\nu>0$ (fixed throughout this paper), external force $f=\operatorname{div} F=\left(\sum_{i=1}^{3} \partial_{i} F_{i, j}\right)_{j=1}^{3}$ and initial value $u_{0}$. First we recall the definition of weak and strong solutions. The space of test functions is defined to be

$$
C_{0}^{\infty}\left(\left[0, T\left[; C_{0, \sigma}^{\infty}(\Omega)\right):=\left\{\left.u\right|_{[0, T[\times \Omega} ; u \in C_{0}^{\infty}(]-1, T[\times \Omega) ; \operatorname{div} u=0\right\} .\right.\right.
$$

Definition 1.1. Let $\Omega \subset \mathbb{R}^{3}$ be an exterior domain and let $u_{0} \in L_{\sigma}^{2}(\Omega)$, $f=\operatorname{div} F$ with $F \in L_{\text {loc }}^{1}\left(\left[0, T\left[; L^{2}(\Omega)\right)\right.\right.$ where $0<T \leq \infty$. Then a vector field $u \in L H_{T}$, where $L H_{T}$ denotes the Leray-Hopf class

$$
L H_{T}:=L_{\mathrm{loc}}^{\infty}\left(\left[0, T\left[; L_{\sigma}^{2}(\Omega)\right) \cap L_{\mathrm{loc}}^{2}\left(\left[0, T\left[; W_{0, \sigma}^{1,2}(\Omega)\right),\right.\right.\right.\right.
$$

is called weak solution (in the sense of Leray-Hopf) of the instationary Navier-Stokes system (1.1) with data $f, u_{0}$, if the following identity is satisfied for all test functions $w \in C_{0}^{\infty}\left(\left[0, T\left[; C_{0, \sigma}^{\infty}(\Omega)\right)\right.\right.$ :

$$
\begin{aligned}
& \int_{0}^{T}\left(-\left\langle u, w_{t}\right\rangle_{\Omega}+\nu\langle\nabla u, \nabla w\rangle_{\Omega}+\langle u \cdot \nabla u, w\rangle_{\Omega}\right) d t \\
& \quad=\left\langle u_{0}, w(0)\right\rangle_{\Omega}-\int_{0}^{T}\langle F, \nabla w\rangle_{\Omega} d t
\end{aligned}
$$

As a consequence of $(1.2),(1.3), u:\left[0, T\left[\rightarrow L_{\sigma}^{2}(\Omega)\right.\right.$ is - after a possible redefinition on a set of Lebesgue measure 0 - weakly continuous and the initial value $u_{0}$ is attained in the sense

$$
\langle u(t), \phi\rangle \rightarrow\left\langle u_{0}, \phi\right\rangle, \quad t \rightarrow 0+\quad \forall \phi \in L_{\sigma}^{2}(\Omega) .
$$

Moreover, there exists a distribution $p$, called an associated pressure, such that the equality

$$
u_{t}-\nu \Delta u+u \cdot \nabla u+\nabla p=f
$$

holds in the sense of distributions on $] 0, T[\times \Omega$, see $[14, \mathrm{~V} .1 .7]$.

A weak solution of (1.1) is called a strong solution if there exist exponents $s, q$ with $2<s<\infty, 3<q<\infty, \frac{2}{s}+\frac{3}{q}=1$ such that additionally Serrin's condition

$$
u \in L^{s}\left(0, T ; L^{q}(\Omega)\right)
$$

is satisfied. By Hölder's inequality, such a strong solution $u$ satisfies $u \otimes u \in$ $L_{\text {loc }}^{2}\left(\left[0, T\left[; L^{2}(\Omega)\right)\right.\right.$. Moreover, by Serrin's Uniqueness Theorem $[14, \mathrm{~V}$. Theorems $1.5 .1,1.4 .1]$, a weak solution with (1.4) is unique within the class of weak solutions satisfying the energy inequality, i.e., fulfilling (1.5) below with $s=0$. Finally, $u:\left[0, T\left[\rightarrow L_{\sigma}^{2}(\Omega)\right.\right.$ is strongly continuous and satisfies the energy identity (1.14) below.

For sufficiently smooth $\Omega, f, u_{0}$ a strong solution $u$ has the regularity property

$$
u \in C^{\infty}(] 0, T[\times \bar{\Omega}), \quad p \in C^{\infty}(] 0, T[\times \bar{\Omega}),
$$


see [14, Theorem V.1.8.2], and therefore a strong solution is also called a regular solution. We call a weak solution $u$ of (1.1) regular at $t$, if there exists a $\delta=\delta(t)>0$ with $u \in L^{s}\left(t-\delta, t+\delta ; L^{q}(\Omega)\right)$ where $s, q$ satisfy $\frac{2}{s}+\frac{3}{q}=1$.

Now let $\Omega \subset \mathbb{R}^{3}$ be an exterior domain with smooth boundary. We know, see [13], that there exists at least one weak solution $u$ of (1.1) satisfying the strong energy inequality

$$
\frac{1}{2}\|u(t)\|_{2}^{2}+\nu \int_{s}^{t}\|\nabla u\|_{2}^{2} d \tau \leq \frac{1}{2}\|u(s)\|_{2}^{2}-\int_{s}^{t}\langle F, \nabla u\rangle_{\Omega} d \tau
$$

for almost all $s \in[0, T[$ and all $t \in[s, T[$.

Our first main theorem states that if $u$ fulfills the Serrin condition in a left-side neighborhood of $t$ then $u$ is regular at $t$. Furthermore, it gives conditions depending on $\|u\|_{L^{s^{\prime}}\left(0, T ; L^{q}(\Omega)\right)}$ with $\frac{2}{s^{\prime}}+\frac{3}{q}>1$ to imply regularity of $u$ at $t$; note that in this case, the integrability of $u$ is weaker than in Serrin's condition.

Theorem 1.2. Let $\Omega \subset \mathbb{R}^{3}$ be an exterior domain with $\partial \Omega \in C^{2,1}$, let $2<$ $s<\infty, \frac{2}{s}+\frac{3}{q}=1, \frac{1}{3}+\frac{1}{q}=\frac{1}{r}$ and let $1 \leq s^{\prime} \leq s$. Assume that $f=\operatorname{div} F$ with $F \in L^{s}\left(0, T ; L^{r}(\Omega)\right) \cap L^{4}\left(0, T ; L^{2}(\Omega)\right), u_{0} \in L_{\sigma}^{2}(\Omega), 0<T<\infty$, and let $u \in L H_{T}$ be a weak solution of the Navier-Stokes equations (1.1) satisfying the strong energy inequality (1.5). Then we have:

1. Left-side $L^{s^{\prime}}\left(L^{q}\right)$-condition. For $\left.t \in\right] 0, T[$ the condition

$$
\liminf _{\delta \rightarrow 0+} \frac{1}{\delta^{1-\frac{s^{\prime}}{s}}} \int_{t-\delta}^{t}\|u(\tau)\|_{q}^{s^{\prime}} d \tau=0
$$

is necessary and sufficient for regularity of $u$ at $t$.

2. Global $L^{s^{\prime}}\left(L^{q}\right)$-condition. There exists a constant $\epsilon_{*}=\epsilon_{*}\left(q, s^{\prime}, \Omega\right)>0$, independent of $f, u_{0}, T$ with the following property: If $u_{0} \in L_{\sigma}^{2}(\Omega) \cap L_{\sigma}^{q}(\Omega)$, $u \in L^{s^{\prime}}\left(0, T ; L_{\sigma}^{q}(\Omega)\right)$ and the conditions

$$
\int_{0}^{T}\|F(\tau)\|_{r}^{s} d \tau \leq \epsilon_{*} \nu^{2 s-1} \quad \text { and } \quad \int_{0}^{T}\|u(\tau)\|_{q}^{s^{\prime}} d \tau \leq \epsilon_{*} \frac{\nu^{s-1}}{\left\|u_{0}\right\|_{q}^{s-s^{\prime}}}
$$

are satisfied, then $u \in L^{s}\left(0, T ; L^{q}(\Omega)\right)$.

Remark. (1) We note that in the case $s^{\prime}=s$ condition (1.6) can be written in the simpler form

$$
u \in L^{s}\left(t-\delta, t ; L_{\sigma}^{q}(\Omega)\right) \text { for some } 0<\delta=\delta(t)<t .
$$

(2) The necessity of (1.6) for a solution $u$ to be regular at $t$ is trivial by Hölder's inequality.

The following theorem states that Hölder continuity of the kinetic energy with exponent $\alpha \in] \frac{1}{2}, 1\left[\right.$ implies regularity of $u$ at $t$. In the case $\alpha=\frac{1}{2}$ we need a smallness condition for the corresponding Hölder term under which we can prove regularity of $u$ at $t$. 
Theorem 1.3. Let $\Omega \subset \mathbb{R}^{3}$ be an exterior domain with boundary $\partial \Omega \in C^{2,1}$, let $0<T<\infty$ and let $u$ be a weak solution of the Navier-Stokes equations (1.1) satisfying the strong energy inequality (1.5) with initial value $u_{0} \in L_{\sigma}^{2}(\Omega)$ and external force $f=\operatorname{div} F$ which will be specified below. Furthermore, we assume that the kinetic energy $E(t):=\frac{1}{2}\|u(t)\|_{2}^{2}$ is a continuous function of $t \in[0, T[$. Then we have:

1. Let $\alpha \in] \frac{1}{2}, 1\left[, 2<s^{\prime}<4 \alpha, 3<q<6, \frac{2}{s^{\prime}}+\frac{3}{q}=\frac{3}{2}, \frac{2}{s}+\frac{3}{q}=1\right.$, let $f \in L^{\frac{s}{s^{\prime}}}\left(0, T ; L^{2}(\Omega)\right)$ and $F \in L^{4}\left(0, T ; L^{2}(\Omega)\right) \cap L^{s}\left(0, T ; L^{r}(\Omega)\right)$, where $\frac{1}{3}+\frac{1}{q}=\frac{1}{r}$, and let $u$ satisfy at $\left.t \in\right] 0, T[$ the left-side condition

$$
\sup _{t-\mu<t^{\prime}<t} \frac{\left|E(t)-E\left(t^{\prime}\right)\right|}{\left|t-t^{\prime}\right|^{\alpha}}<\infty
$$

with $a \mu>0$. Then $u$ is regular at $t$.

2. (The case $\left.\alpha=\frac{1}{2}\right)$ Let $f \in L^{2}\left(0, T ; L^{2}(\Omega)\right), F \in L^{4}\left(0, T ; L^{2}(\Omega)\right)$. Then there exists a constant $\gamma_{*}=\gamma_{*}(\Omega)$ such that the left-side condition

$$
\sup _{t-\mu<t^{\prime}<t} \frac{\left|E(t)-E\left(t^{\prime}\right)\right|}{\left|t-t^{\prime}\right|^{\frac{1}{2}}} \leq \gamma_{*} \nu^{\frac{5}{2}}
$$

with a $\mu>0$ implies regularity of $u$ at $t$.

Remark. (1) The proof of Theorem 1.3, in particular see (4.8), will yield the following regularity criteria using the dissipation energy: If

$$
\alpha \in] \frac{1}{2}, 1\left[\text { and } \quad \liminf _{\delta \rightarrow 0+} \frac{1}{\delta^{\alpha}} \int_{t-\delta}^{t}\|\nabla u(\tau)\|_{2}^{2} d \tau<\infty\right.
$$

or

$$
\liminf _{\delta \rightarrow 0+} \frac{1}{\delta^{\frac{1}{2}}} \int_{t-\delta}^{t}\|\nabla u(\tau)\|_{2}^{2} d \tau \leq \gamma_{*} \nu^{\frac{3}{2}}
$$

then $u$ is regular at $t$.

(2) In the case $\alpha=\frac{1}{2}$ a smallness condition as in (1.9) and (1.11) is necessary. Indeed, if $f=0$ and $] 0, t$ [ is a maximal regularity interval of $u$, then

$$
\|\nabla u(\tau)\|_{2} \geq \frac{c_{0}}{(t-\tau)^{\frac{1}{4}}}, \quad 0<\tau<t,
$$

where $c_{0}=c_{0}(\Omega)>0$, see $[8]$. Hence

$$
\liminf _{\delta \rightarrow 0+} \frac{1}{\delta^{\frac{1}{2}}} \int_{t-\delta}^{t}\|\nabla u(\tau)\|_{2}^{2} d \tau \geq 2 c_{0}^{2}>0
$$

and due to the strong energy inequality (1.5) it holds for all $\mu>0$

$$
\sup _{t-\mu<t^{\prime}<t} \frac{\left|E(t)-E\left(t^{\prime}\right)\right|}{\left|t-t^{\prime}\right|^{\frac{1}{2}}} \geq 2 \nu c_{0}^{2}>0 .
$$

The proofs of the regularity criteria formulated in this paper are based on a local or global identification of a weak solution with a very weak solution, a concept described in Definition 2.3 below. The following key result, Theorem 1.4, gives conditions under which a given very weak solution is also a weak solution in the sense of Leray-Hopf and, therefore, yields under certain 
smallness conditions on the data $f$ and $u_{0}$ the existence of a unique strong solution of $(1.1)$ on $[0, T[\times \Omega$.

Theorem 1.4. Let $\Omega \subset \mathbb{R}^{3}$ be an exterior domain with $\partial \Omega \in C^{2,1}$, let $2<s<\infty$, $\frac{2}{s}+\frac{3}{q}=1$ and let $\frac{1}{3}+\frac{1}{q}=\frac{1}{q_{*}}$. Then there exists a constant $\epsilon_{*}=\epsilon_{*}(q, \Omega)>0$ with the following property: Given $0<T<\infty$ and data $u_{0} \in L_{\sigma}^{2}(\Omega) \cap L_{\sigma}^{q}(\Omega)$ and $f=\operatorname{div} F$ with $F \in L^{s}\left(0, T ; L^{q *}(\Omega)\right) \cap L^{4}\left(0, T ; L^{2}(\Omega)\right)$ satisfying the following two conditions:

$$
\begin{gathered}
\int_{0}^{T}\|F(\tau)\|_{q_{*}}^{s} d \tau \leq \epsilon_{*} \nu^{2 s-1}, \\
\int_{0}^{T}\left\|e^{-\nu \tau A_{q}} u_{0}\right\|_{q}^{s} d \tau \leq \epsilon_{*} \nu^{s-1} .
\end{gathered}
$$

In this case, there exists a unique weak solution $u \in L H_{T}$ of (1.1) satisfying the Serrin condition $u \in L^{s}\left(0, T ; L^{q}(\Omega)\right)$. After a possible redefinition on a set of Lebesgue measure 0 , we get that $u:\left[0, T\left[\rightarrow L_{\sigma}^{2}(\Omega)\right.\right.$ is strongly continuous and it holds the energy identity

$$
\frac{1}{2}\|u(t)\|_{2}^{2}+\nu \int_{0}^{t}\|\nabla u\|_{2}^{2} d \tau=\frac{1}{2}\left\|u_{0}\right\|_{2}^{2}-\int_{0}^{t}\langle F, \nabla u\rangle_{\Omega} d \tau
$$

for all $t \in[0, T[$.

The proof of this crucial result is the content of Sect. 3 and differs from the case of bounded domains, see [4,6], where the trivial inclusion $L^{q}(\Omega) \subset L^{r}(\Omega)$, $q>r$, yielding also better embedding properties of fractional powers of the Stokes operator $A_{q}$, was used several times. The main idea of the proof is to construct a very weak solution $v \in L^{s}\left(0, T ; L_{\sigma}^{q}(\Omega)\right)$ for the given data $u_{0}, f$ and to identify $u$ and $v$ by Serrin's Uniqueness Theorem; for this reason, we have to show that $v$ lies in the Leray-Hopf class $L H_{T}$.

After some preliminaries to be discussed in Sect. 2 we prove Theorem 1.4 in Sect. 3. Finally, Sect. 4 deals with the proofs of the main results Theorem 1.2 and 1.3 .

\section{Preliminaries}

Given $1 \leq q \leq \infty, k \in \mathbb{N}$ we need the usual Lebesgue and Sobolev spaces, $L^{q}(\Omega), W^{k, q}(\Omega)$ with norm $\|\cdot\|_{L^{q}(\Omega)}=\|\cdot\|_{q}$ and $\|\cdot\|_{W^{k, q}(\Omega)}=\|\cdot\|_{k, q}$, respectively. For two measurable functions $f, g$ with the property $f \cdot g \in L^{1}(\Omega)$, where $f \cdot g$ means the usual scalar product of vector or matrix fields, we set

$$
\langle f, g\rangle_{\Omega}:=\int_{\Omega} f(x) \cdot g(x) d x .
$$

Note that the same symbol $L^{q}(\Omega)$ etc. will be used for spaces of scalar-, vector or matrix-valued functions. Let $C^{m}(\Omega), m=0,1, \ldots, \infty$, denote the usual space of functions for which all partial derivatives of order $|\alpha| \leq m$ exist and are continuous. As usual, $C_{0}^{m}(\Omega)$ is the set of all functions from $C^{m}(\Omega)$ with 
compact support in $\Omega$. Further we need the space of smooth solenoidal vector fields

$$
C_{0, \sigma}^{\infty}(\Omega):=\left\{v \in C_{0}^{\infty}(\Omega)^{3} ; \operatorname{div} v=0\right\}
$$

and define the spaces

$$
L_{\sigma}^{q}(\Omega):={\overline{C_{0, \sigma}^{\infty}(\Omega)}}^{\|\cdot\|_{q}}, \quad W_{0, \sigma}^{1,2}(\Omega):={\overline{C_{0, \sigma}^{\infty}(\Omega)}}^{\|\cdot\|_{W^{1,2}}} .
$$

For $1 \leq q \leq \infty$ let $q^{\prime} \in[1, \infty]$ denote its dual exponent. It is well known that $L_{\sigma}^{q}(\Omega)^{\prime}=L_{\sigma}^{q^{\prime}}(\Omega)$ using the standard pairing $\langle\cdot, \cdot\rangle_{\Omega}$. Moreover, let us write $[d, v]_{\Omega}$ for the application of a distribution $d \in C_{0}^{\infty}(\Omega)^{\prime}$ on a test function $v \in C_{0}^{\infty}(\Omega)$.

Given a Banach space $X$ and an interval $[0, T], 0<T \leq \infty$, we denote by $L^{p}(0, T ; X), 1 \leq p \leq \infty$, the space of all equivalence classes of strongly measurable functions $f:[0, T) \rightarrow X$ such that

$$
\|f\|_{p}:=\left(\int_{0}^{T}\|f(t)\|_{X}^{p} d t\right)^{\frac{1}{p}}<\infty
$$

if $p<\infty$, and $\|f\|_{\infty}:=\operatorname{ess} \sup _{[0, T[}\|f(\cdot)\|_{X}$, if $p=\infty$. Moreover, we define the set of locally integrable $L^{p}$-functions on $[0, T[$ as

$$
\begin{aligned}
L_{\text {loc }}^{p}([0, T[; X):= & \{u:[0, T[\rightarrow X \text { strongly measurable, } \\
& \left.u \in L^{p}\left(0, T^{\prime} ; X\right) \text { for all } 0<T^{\prime}<T\right\} .
\end{aligned}
$$

When $X=L^{q}(\Omega), 1 \leq q \leq \infty$, we denote the norm in $L^{p}\left(0, T ; L^{q}(\Omega)\right)$ by $\|\cdot\|_{q, p, \Omega ; T}$. For $1<p, q<\infty$ it holds

$$
L^{p}\left(0, T ; L^{q}(\Omega)\right)^{\prime}=L^{p^{\prime}}\left(0, T ; L^{q^{\prime}}(\Omega)\right)
$$

and we define

$$
\langle f, g\rangle_{\Omega, T}:=\int_{0}^{T} \int_{\Omega} f(t, x) \cdot g(t, x) d x d t
$$

for $f \in L^{p}\left(0, T ; L^{q}(\Omega)\right), g \in L^{p^{\prime}}\left(0, T ; L^{q^{\prime}}(\Omega)\right)$.

Given an exterior domain $\Omega \subset \mathbb{R}^{3}$ with $\partial \Omega \in C^{2,1}$ and $1<q<\infty$, there exists a bounded, linear projection $P_{q}: L^{q}(\Omega) \rightarrow L_{\sigma}^{q}(\Omega)$ with range $\mathcal{R}\left(P_{q}\right)=L_{\sigma}^{q}(\Omega)$ and nullspace $N\left(P_{q}\right)=\left\{\nabla p \in L^{q}(\Omega) ; p \in L_{\text {loc }}^{q}(\bar{\Omega})\right\}$. The operator $P_{q}$ is called Helmholtz projection and is consistent in the sense that

$$
P_{q} f=P_{r} f \quad \forall f \in L^{q}(\Omega) \cap L^{r}(\Omega) .
$$

Furthermore, we get $P_{q}^{\prime}=P_{q^{\prime}}$ for the dual operator, i.e.,

$$
\left\langle P_{q} f, g\right\rangle_{\Omega}=\left\langle f, P_{q^{\prime}} g\right\rangle_{\Omega} \quad \forall f \in L^{q}(\Omega) \quad \forall g \in L^{q^{\prime}}(\Omega) .
$$

For $1<q<\infty$ we define the Stokes operator $A_{q}$ on $L_{\sigma}^{q}(\Omega)$ by

$$
\begin{aligned}
\mathcal{D}\left(A_{q}\right) & =L_{\sigma}^{q}(\Omega) \cap W_{0}^{1, q}(\Omega) \cap W^{2, q}(\Omega), \\
A_{q} u & :=-P_{q} \Delta u, \quad u \in \mathcal{D}\left(A_{q}\right) .
\end{aligned}
$$

The Stokes operator is consistent in the sense that for $1<q, r<\infty$ it holds

$$
A_{q} u=A_{r} u \quad \forall u \in \mathcal{D}\left(A_{q}\right) \cap \mathcal{D}\left(A_{r}\right) .
$$


In general, $\mathcal{D}\left(A_{q}\right)$ will be equipped with the graph norm $\|u\|_{\mathcal{D}\left(A_{q}\right)}:=\|u\|_{q}+$ $\left\|A_{q}\right\|_{q}$ for $u \in D\left(A_{q}\right)$ which makes $\mathcal{D}\left(A_{q}\right)$ to a Banach space since the Stokes operator is closed. For simplicity, we use the notation $A=A_{2}$.

For $\alpha \in[-1,1]$ the fractional power $A_{q}^{\alpha}: \mathcal{D}\left(A_{q}^{\alpha}\right) \rightarrow L_{\sigma}^{q}(\Omega)$ with dense domain $\left.\mathcal{D}\left(A_{q}^{\alpha}\right) \subseteq L_{\sigma}^{q}(\Omega)\right)$ is a well defined, injective, closed operator such that

$$
\left(A_{q}^{\alpha}\right)^{-1}=A_{q}^{-\alpha}, \quad \mathcal{R}\left(A_{q}^{\alpha}\right)=\mathcal{D}\left(A_{q}^{-\alpha}\right) \quad \text { and } \quad\left(A_{q}^{\alpha}\right)^{\prime}=A_{q^{\prime}}^{\alpha} .
$$

It holds $\mathcal{D}\left(A_{q}^{1 / 2}\right)=W_{0}^{1, q}(\Omega) \cap L_{\sigma}^{q}(\Omega)$ for $1<q<3$, and the estimate

$$
\|\nabla u\|_{q, \Omega} \leq c\left\|A_{q}^{1 / 2} u\right\|_{q, \Omega} \quad \text { for } 1<q<3, u \in \mathcal{D}\left(A_{q}^{1 / 2}\right),
$$

with a constant $c=c(\Omega, q)>0$. Moreover,

$$
\|u\|_{\gamma, \Omega} \leq c\left\|A_{q}^{\alpha} u\right\|_{q, \Omega} \quad \text { where } 0 \leq \alpha \leq \frac{1}{2}, 1<q<3,2 \alpha+\frac{3}{\gamma}=\frac{3}{q},
$$

for all $u \in \mathcal{D}\left(A_{q}^{\alpha}\right)$ with a constant $c=c(\Omega, q, \gamma)>0$. It is well known that $-A_{q}$ generates a uniformly bounded analytic semigroup $\left\{e^{-t A_{q}}: t \geq 0\right\}$ on $L_{\sigma}^{q}(\Omega)$ satisfying the decay estimate

$$
\left\|A_{q}^{\alpha} e^{-t A_{q}}\right\|_{q} \leq c t^{-\alpha} \quad \forall t>0
$$

where $\alpha \geq 0,1<q<\infty$ and $c=c(\Omega, q, \alpha)>0$. We refer to [7,9-11] for these properties of the Stokes operator in exterior domains.

Lemma 2.1. Let $d \in C_{0}^{\infty}(\Omega)^{\prime}$ be a distribution, well defined for all $v \in \mathcal{D}\left(A_{q^{\prime}}^{\alpha}\right)$ where $1<q<\infty, 0<\alpha \leq 1$. We assume that there exists a constant $c \geq 0$, independent of $v \in \mathcal{D}\left(A_{q^{\prime}}^{\alpha}\right)$, such that

$$
\left|[d, v]_{\Omega}\right| \leq c\left\|A_{q^{\prime}}^{\alpha} v\right\|_{q^{\prime}, \Omega} \quad \forall v \in \mathcal{D}\left(A_{q^{\prime}}^{\alpha}\right) .
$$

Then there exists a unique element $\tilde{d} \in L_{\sigma}^{q}(\Omega)$, to be denoted by $A_{q}^{-\alpha} P_{q} d$, with the properties

$$
\left\langle A_{q}^{-\alpha} P_{q} d, A_{q^{\prime}}^{\alpha} v\right\rangle_{\Omega}=[d, v]_{\Omega} \quad \forall v \in \mathcal{D}\left(A_{q^{\prime}}^{\alpha}\right) \quad \text { and }\left\|A_{q}^{-\alpha} P_{q} d\right\|_{q} \leq c
$$

with the constant $c$ from (2.9). In particular, if $F \in L^{q}(\Omega)$, and $\frac{3}{2}<q<\infty$, then $A_{q}^{-\frac{1}{2}} P_{q} \operatorname{div} F \in L_{\sigma}^{q}(\Omega)$ and

$$
\left\|A_{q}^{-\frac{1}{2}} P_{q} \operatorname{div} F\right\|_{q} \leq c\|F\|_{q}
$$

Proof. We define for $w \in \mathcal{R}\left(A_{q^{\prime}}^{\alpha}\right)$

$$
[\widetilde{d}, w]_{\Omega}:=[d, v]_{\Omega}, \quad \text { where } w=A_{q^{\prime}}^{\alpha} v, v \in \mathcal{D}\left(A_{q^{\prime}}^{\alpha}\right) .
$$

By the density of $\mathcal{R}\left(A_{q^{\prime}}^{\alpha}\right)$ in $L_{\sigma}^{q^{\prime}}(\Omega)$, we extend $\tilde{d}$ to a functional defined on $L_{\sigma}^{q^{\prime}}(\Omega)$. We use $L_{\sigma}^{q^{\prime}}(\Omega)^{\prime}=L_{\sigma}^{q}(\Omega)$ to obtain a unique element $A_{q}^{-\alpha} P_{q} d \in L_{\sigma}^{q}(\Omega)$ satisfying the identity in (2.10). For the proof of (2.11) we exploit (2.6) with $q$ replaced by $\left.q^{\prime} \in\right] 1,3[$. 
Theorem 2.2. Let $\Omega \subset \mathbb{R}^{3}$ be an exterior domain with $\partial \Omega \in C^{2,1}$, let $1<q, s<$ $\infty$ and $0<T<\infty$. Furthermore, let $f \in L^{s}\left(0, T ; L_{\sigma}^{q}(\Omega)\right)$ and $u_{0} \in L_{\sigma}^{q}(\Omega)$ such that $\int_{0}^{\infty}\left\|A_{q} e^{-t A_{q}} u_{0}\right\|_{q, \Omega}^{s} d t<\infty$. Then the instationary Stokes system

$$
\begin{aligned}
u_{t}+\nu A_{q} u & =f \quad \text { in }(0, T) \\
u(0) & =u_{0}
\end{aligned}
$$

has a unique strong solution $u \in L^{s}\left(0, T ; D\left(A_{q}\right)\right)$ with $u_{t} \in L^{s}\left(0, T ; L_{\sigma}^{q}(\Omega)\right)$ and $u \in C\left(\left[0, T\left[; L_{\sigma}^{q}(\Omega)\right)\right.\right.$. Moreover, $u$ satisfies the maximal regularity estimate

$$
\begin{aligned}
& \left\|u_{t}\right\|_{q, s, \Omega ; T}+\left\|\nu A_{q} u\right\|_{q, s, \Omega ; T} \\
& \quad \leq c\left(\left(\int_{0}^{T}\left\|\nu A_{q} e^{-\nu t A_{q}} u_{0}\right\|_{q, \Omega}^{s} d t\right)^{\frac{1}{s}}+\|f\|_{q, s, \Omega ; T}\right)
\end{aligned}
$$

with a constant $c=c(\Omega, q, s)$ independent of $T$ and $\nu$. It holds the representation

$$
u(t)=e^{-\nu t A_{q}} u_{0}+\int_{0}^{t} e^{-\nu(t-\tau) A_{q}} f(\tau) d \tau
$$

for all $t \in[0, T[$. In the case $T=\infty$ we get a unique strong solution $u \in L_{\mathrm{loc}}^{s}\left(0, \infty ; D\left(A_{q}\right)\right)$ of $(2.12)$ satisfying $u_{t} \in L^{s}\left(0, \infty ; L_{\sigma}^{q}(\Omega)\right)$ and $u \in$ $C\left(\left[0, \infty\left[; L_{\sigma}^{q}(\Omega)\right)\right.\right.$ and it holds the estimate (2.13) and the representation $(2.14)$ for all $t \in[0, \infty[$.

Proof. See [10, Theorem 4.2].

A major tool for the proof of Theorem 1.4 is the theory of very weak solutions. In this context we refer to [3] for exterior domains and to [4] for bounded domains. In the following definition let

$$
\begin{aligned}
& C_{0}^{1}\left(\left[0, T\left[; C_{0, \sigma}^{2}(\bar{\Omega})\right):=\left\{\left.w\right|_{[0, T[\times \bar{\Omega}} \text { with } w \in C_{0}^{1,2}(-] 1, T\left[\times \mathbb{R}^{3}\right) ;\right.\right.\right. \\
& \operatorname{div} w=0,\left.w\right|_{\partial \Omega}=0 \text { for all } t \in[0, T[\}
\end{aligned}
$$

denote the space of test functions and let

$$
\begin{aligned}
\mathcal{J}^{q, s}(\Omega):=\{ & u_{0} \in C_{0}^{\infty}(\Omega)^{\prime} ; \\
& \left.A_{q}^{-1} P_{q} u_{0} \in L_{\sigma}^{q}(\Omega), \int_{0}^{\infty}\left\|A_{q} e^{-t A_{q}}\left(A_{q}^{-1} P_{q} u_{0}\right)\right\|_{q, \Omega}^{s} d t<\infty\right\}
\end{aligned}
$$

denote the space of initial values.

Definition 2.3. Let $\Omega \subset \mathbb{R}^{3}$ be an exterior domain, let $F \in L^{s}\left(0, T ; L^{r}(\Omega)\right)$ and $u_{0} \in \mathcal{J}^{q, s}(\Omega)$ where $2<s<\infty, \frac{2}{s}+\frac{3}{q}=1, \frac{1}{3}+\frac{1}{q}=\frac{1}{r}$. Then $u \in L^{s}\left(0, T ; L_{\sigma}^{q}(\Omega)\right)$ is called very weak solution of the instationary Navier-Stokes equations (1.1) if

$$
\int_{0}^{T}\left\langle-u, w_{t}\right\rangle_{\Omega}-\nu\langle u, \Delta w\rangle_{\Omega}-\langle u \otimes u, \nabla w\rangle_{\Omega} d t=\left[u_{0}, w(0)\right]_{\Omega}-\int_{0}^{T}\langle F, \nabla w\rangle_{\Omega} d t
$$

holds for all test functions $w \in C_{0}^{1}\left(\left[0, T\left[; C_{0, \sigma}^{2}(\bar{\Omega})\right)\right.\right.$. 
In the corresponding definition of very weak solutions to the linear, instationary Stokes system where the nonlinear term $u \cdot \nabla u$ is absent, we may omit in Definition 2.3 the restriction $\frac{2}{s}+\frac{3}{q}=1$, and in (2.15) the term $\langle u \otimes u, \nabla w\rangle_{\Omega, T}$ is absent. A proof of the following Theorem can be found in $[3,12]$.

Theorem 2.4. Let $\Omega \subset \mathbb{R}^{3}$ be an exterior domain with $\partial \Omega \in C^{2,1}$ and let $2<s<\infty, \frac{2}{s}+\frac{3}{q}=1, \frac{1}{3}+\frac{1}{q}=\frac{1}{r}$. Then there exists a constant $c=c(q, \Omega)>0$ with the following property: For data $f=\operatorname{div} F$ with $F \in L^{s}\left(0, T ; L^{r}(\Omega)\right)$ and $u_{0} \in \mathcal{J}^{q, s}(\Omega)$, satisfying the condition

$$
\left(\int_{0}^{T}\left\|\nu A_{q} e^{-\nu t A_{q}}\left(A_{q}^{-1} P_{q} u_{0}\right)\right\|_{q, \Omega}^{s} d t\right)^{\frac{1}{s}}+\|F\|_{r, s, \Omega ; T} \leq c \nu^{1+\alpha}
$$

with $\alpha:=\frac{3}{2 q}+\frac{1}{2}=1-\frac{1}{s}$, there exists a unique very weak solution $u \in$ $L^{s}\left(0, T ; L_{\sigma}^{q}(\Omega)\right)$ of the instationary Navier-Stokes system (1.1). Moreover, $u$ has the representation $u=E+\tilde{u}$, where $E \in L^{s}\left(0, T ; L_{\sigma}^{q}(\Omega)\right)$ is the unique very weak solution of the linear Stokes system with data $f, u_{0}$ and $\tilde{u}$ is the unique solution in $L^{s}\left(0, T ; L_{\sigma}^{q}(\Omega)\right)$ of the nonlinear fixed point equation

$$
\tilde{u}(t)=-\int_{0}^{t} A_{q}^{\alpha} e^{-\nu(t-\tau) A_{q}} A_{q}^{-\alpha} P_{q} \operatorname{div}((\tilde{u}(\tau)+E(\tau)) \otimes(\tilde{u}(\tau)+E(\tau))) d \tau
$$

for almost all $t \in[0, T[$.

Finally we recall the Hardy-Littlewood inequality [14, II Lemma 3.3.2]. Let $0<\alpha<1,1<r<q<\infty$ with $\alpha+\frac{1}{q}=\frac{1}{r}$ and let $f \in L^{r}(\mathbb{R})$. Then the integral

$$
u(t):=\int_{\mathbb{R}}|t-\tau|^{\alpha-1} f(\tau) d \tau
$$

converges absolutely for almost all $t \in \mathbb{R}$ and it holds

$$
\|u\|_{L^{q}(\mathbb{R})} \leq c\|f\|_{L^{r}(\mathbb{R})}
$$

with a constant $c=c(\alpha, q)>0$.

\section{Proof of Theorem 1.4}

Before proving Theorem 1.4 we discuss the nonlinear term arising in the nonlinear fixed point problem (2.17). We denote by $\operatorname{div}(u \otimes u)$ the functional defined for suitable vector fields $w$ by

$$
[\operatorname{div}(u \otimes u), w]_{\Omega}:=-\langle u \otimes u, \nabla w\rangle_{\Omega} .
$$

The following lemma is technical but essential for Lemma 3.2 below.

Lemma 3.1. Let $\Omega \subset \mathbb{R}^{3}$ be an exterior domain with boundary $\partial \Omega \in C^{2,1}$, let $3<q<\infty, r \in\left[\frac{q}{2}, q\right]$ and $\beta:=\frac{3}{q}-\frac{3}{2 r}+\frac{1}{2}$.

1. There exists a constant $c=c(\Omega, q, r)>0$ such that for all $u \in L_{\sigma}^{q}(\Omega)$

$$
\left\|A_{r}^{-\beta} P_{r} \operatorname{div}(u \otimes u)\right\|_{r, \Omega} \leq c\|u\|_{q, \Omega}^{2} .
$$


2. For $2<s<\infty, 3<q<\infty, 0<T \leq \infty$ there exists a constant $c=$ $c(\Omega, q, r)>0$ such that for all $u \in L^{s}\left(0, T ; L_{\sigma}^{q}(\Omega)\right)$

$$
\left\|A_{r}^{-\beta} P_{r} \operatorname{div}(u \otimes u)\right\|_{r, \frac{s}{2}, \Omega ; T} \leq c\|u\|_{q, s, \Omega ; T}^{2} .
$$

Proof. The assumptions of the lemma imply

$$
2\left(\beta-\frac{1}{2}\right)+\frac{3}{\left(\frac{q}{2}\right)^{\prime}}=\frac{3}{r^{\prime}} \quad \text { with } 1<r^{\prime}<3, \frac{1}{2} \leq \beta<1 .
$$

Then we get for arbitrary $w \in \mathcal{D}\left(A_{r^{\prime}}^{\beta}\right)$ by (2.6) using $1<\left(\frac{q}{2}\right)^{\prime}<3$, (2.7) and (2.5) (applied to $A^{1 / 2}$ instead of $A$ )

$$
\begin{aligned}
|[\operatorname{div}(u \otimes u), w]| & =|-\langle u \otimes u, \nabla w\rangle| \\
& \leq\|u \otimes u\|_{\frac{q}{2}}\|\nabla w\|_{\left(\frac{q}{2}\right)^{\prime}} \\
& \leq c\|u\|_{q}^{2}\left\|A_{(q / 2)^{\prime}}^{1 / 2} w\right\|_{\left(\frac{q}{2}\right)^{\prime}} \\
& \leq c\|u\|_{q}^{2}\left\|A_{r^{\prime}}^{\left(\beta-\frac{1}{2}\right)}\left(A_{(q / 2)^{\prime}}^{1 / 2} w\right)\right\|_{r^{\prime}} \\
& \leq c\|u\|_{q}^{2}\left\|A_{r^{\prime}}^{\beta} w\right\|_{r^{\prime}} .
\end{aligned}
$$

It is possible to choose the constant $c>0$ in the above estimate depending only on $\Omega, q$ and $r$. For the second assertion we use (3.1), which holds for almost all $t \in[0, T[$, and integrate over $[0, T]$.

Lemma 3.2. Let $\Omega \subset \mathbb{R}^{3}$ be an exterior domain with $\partial \Omega \in C^{2,1}$, let $0<T \leq \infty$, $2<s<\infty, \frac{2}{s}+\frac{3}{q}=1$ and let $u \in L^{s}\left(0, T ; L^{q}(\Omega)\right)$. We define for $r \in\left[\frac{q}{2}, q\right]$ and $\beta:=\frac{3}{q}-\frac{3}{2 r}+\frac{1}{2}$ the term $\Lambda^{r}(u)$ by

$$
\Lambda^{r} u(t):=-\int_{0}^{t} A_{r}^{\beta} e^{-\nu(t-\tau) A_{r}} A_{r}^{-\beta} P_{r} \operatorname{div}(u(\tau) \otimes u(\tau)) d \tau .
$$

Then the following statements are satisfied.

1. For almost all $t \in[0, T[$ we get

$$
\int_{0}^{t}\left\|A_{r}^{\beta} e^{-\nu(t-\tau) A_{r}} A_{r}^{-\beta} P_{r} \operatorname{div}(u(\tau) \otimes u(\tau))\right\|_{r} d \tau<\infty
$$

and

$$
\begin{aligned}
& -A_{r}^{\beta} \int_{0}^{t} e^{-\nu(t-\tau) A_{r}} A_{r}^{-\beta} P_{r} \operatorname{div}(u(\tau) \otimes u(\tau)) d \tau \\
& =-\int_{0}^{t} A_{r}^{\beta} e^{-\nu(t-\tau) A_{r}} A_{r}^{-\beta} P_{r} \operatorname{div}(u(\tau) \otimes u(\tau)) d \tau .
\end{aligned}
$$

2. For all $r_{1}, r_{2} \in\left[\frac{q}{2}, q\right]$ with $\beta_{1}:=\frac{3}{q}-\frac{3}{2 r_{1}}+\frac{1}{2}, \beta_{2}:=\frac{3}{q}-\frac{3}{2 r_{2}}+\frac{1}{2}$ it holds

$$
\Lambda^{r_{1}} u(t)=\Lambda^{r_{2}} u(t) \quad \text { for almost all } t \in[0, T[.
$$

Therefore, we can denote the expression in (3.4), independently of $r \in$ $\left[\frac{q}{2}, q\right]$, by $\Lambda(u)$. 
3. For all $q_{1} \in\left[\frac{q}{2}, q\right]$ with $3<q_{1}<\infty$ and $s_{1}>2$ defined by $\frac{2}{s_{1}}+\frac{3}{q_{1}}=1$ we have

$$
\Lambda u \in L^{s_{1}}\left(0, T ; L^{q_{1}}(\Omega)\right)
$$

4. If $q \in] 3,6[$ then

$$
\Lambda u \in L^{\frac{s}{2}}\left(0, T ; L^{q_{2}}(\Omega)\right)
$$

where $q_{2}>3$ satisfies $\frac{1}{3}+\frac{1}{q_{2}}=\frac{1}{\left(\frac{q}{2}\right)}$ and consequently $\frac{2}{\left(\frac{s}{2}\right)}+\frac{3}{q_{2}}=1$.

Proof. (1) By (2.8) and (3.1) we know that for all $t \in[0, T[$

$$
\begin{gathered}
\int_{0}^{t}\left\|A_{r}^{\beta} e^{-\nu(t-\tau) A_{r}} A_{r}^{-\beta} P_{r} \operatorname{div}(u(\tau) \otimes u(\tau))\right\|_{r} d \tau \\
\leq c(\Omega, q, r) \nu^{-\beta} \int_{0}^{T}|t-\tau|^{-\beta}\|u(\tau)\|_{q}^{2} d \tau .
\end{gathered}
$$

Moreover, as for almost all $t \in[0, T$ [ the integral in (3.10) is finite (see the application of the Hardy-Littlewood inequality (2.18) in the proof of part (3) below) and

$$
\int_{0}^{t}\left\|e^{-\nu(t-\tau) A_{r}} A_{r}^{-\beta} P_{r} \operatorname{div}(u \otimes u)\right\|_{r} d \tau \leq c \int_{0}^{t}\left\|A_{r}^{-\beta} P_{r} \operatorname{div}(u \otimes u)\right\|_{r} d \tau<\infty,
$$

the closedness of the operator $A_{r}^{\beta}$ yields the identity (3.6).

(2) To prove (3.7) for $t \in(0, T[$ as in (1) let

$$
\left.f_{t}^{r}(\tau):=A_{r}^{\beta} e^{-\nu(t-\tau) A_{r}} A_{r}^{-\beta} P_{r} \operatorname{div}(u(\tau) \otimes u(\tau)) \quad \text { for almost all } \tau \in\right] 0, t[,
$$

where $\beta=\beta(r)=\frac{3}{q}-\frac{3}{2 r}+\frac{1}{2}$. Since for all $\phi \in C_{0, \sigma}^{\infty}(\Omega)$

$$
\int_{0}^{t}\left\langle f_{t}^{r_{1}}(\tau), \phi\right\rangle_{\Omega} d \tau=-\int_{0}^{t}\left\langle u(\tau) \otimes u(\tau), \nabla e^{\left.-\nu(t-\tau) A_{r^{\prime}} \phi\right\rangle_{\Omega}} d \tau,\right.
$$

we see that

$$
\int_{0}^{t}\left\langle f_{t}^{r_{1}}(\tau), \phi\right\rangle_{\Omega} d \tau=\int_{0}^{t}\left\langle f_{t}^{r_{2}}(\tau), \phi\right\rangle_{\Omega} d \tau
$$

for details of the proof we refer to [12]. A density argument finishes the proof of (3.7).

(3) We consider (3.10) and use the Hardy-Littlewood inequality (2.18) with $(1-\beta)+\frac{1}{s_{1}}=\frac{1}{\left(\frac{s}{2}\right)}$ to conclude with $\Lambda^{q_{1}} u=\Lambda u$ and (3.2) that

$$
\begin{aligned}
& \|\Lambda u\|_{q_{1}, s_{1}, \Omega ; T} \\
& \quad \leq\left(\int_{0}^{T}\left(c \nu^{-\beta} \int_{0}^{T}|t-\tau|^{-\beta}\left\|A_{q_{1}}^{-\beta} P_{q_{1}} \operatorname{div}(u(\tau) \otimes u(\tau))\right\|_{q_{1}} d \tau\right)^{s_{1}} d t\right)^{\frac{1}{s_{1}}} \\
& \quad \leq c \nu^{-\beta}\left\|A_{q_{1}}^{-\beta} P_{q_{1}} \operatorname{div}(u(\tau) \otimes u(\tau))\right\|_{q_{1}, \frac{s}{2}, \Omega ; T} \\
& \quad \leq c\left(q, q_{1}, \Omega\right) \nu^{-\beta}\|u\|_{q, s, \Omega ; T}^{2}<\infty
\end{aligned}
$$


(4) From $2 \frac{1}{2}+\frac{3}{q_{2}}=\frac{3}{\left(\frac{q}{2}\right)}$ and (2.7) it follows with (3.6) and $\beta=\frac{1}{2}, r=\frac{q}{2}$, for almost all $t \in[0, T[$

$$
\begin{aligned}
\left\|\Lambda^{q_{2}} u(t)\right\|_{q_{2}} & \leq\left\|A_{\frac{q}{2}}^{1 / 2} \Lambda u(t)\right\|_{\frac{q}{2}} \\
& =\left\|A_{\frac{q}{2}} \int_{0}^{t} e^{-\nu(t-\tau) A_{\frac{q}{2}}} A_{\frac{q}{2}}^{-1 / 2} P_{\frac{q}{2}} \operatorname{div}(u(\tau) \otimes u(\tau)) d \tau\right\|_{\frac{q}{2}} .
\end{aligned}
$$

Since by $(3.2)$

$$
A_{\frac{q}{2}}^{-1 / 2} P_{\frac{q}{2}} \operatorname{div}(u \otimes u) \in L^{\frac{s}{2}}\left(0, T ; L^{\frac{q}{2}}(\Omega)\right),
$$

the maximal regularity estimate (2.13) yields the last statement of the lemma.

Proof of Theorem 1.4. Given the smallness conditions (1.12) and (1.13), there exists by Theorem 2.4 a unique very weak solution $u \in L^{s}\left(0, T ; L_{\sigma}^{q}(\Omega)\right)$ of (1.1). Moreover, we know the representation $u=E+\tilde{u}$, where the linear part $E$ satisfies

$$
E(t)=e^{-\nu t A_{q}} u_{0}+A_{q} \int_{0}^{t} e^{-\nu(t-\tau) A_{q}}\left(A_{q}^{-1} P_{q} \operatorname{div} F(\tau)\right) d \tau
$$

in $\left[0, T\left[\right.\right.$ and the nonlinear part $\tilde{u} \in L^{s}\left(0, T ; L_{\sigma}^{q}(\Omega)\right)$ solves the fixed point equation

$$
\tilde{u}(t)=-\int_{0}^{t} A_{q}^{\alpha} e^{-\nu(t-\tau) A_{q}} A_{q}^{-\alpha} P_{q} \operatorname{div}((\tilde{u}(\tau)+E(\tau)) \otimes(\tilde{u}(\tau)+E(\tau))) d \tau
$$

with $\alpha:=\frac{3}{2 q}+\frac{1}{2}$ for almost all $t \in\left[0, T\left[\right.\right.$. Since $F \in L^{2}\left(0, T ; L^{2}(\Omega)\right)$ and $u_{0} \in L_{\sigma}^{2}(\Omega)$ it follows with $(2.5)$ that

$$
E(t)=E_{1}(t)+E_{2}(t):=e^{-\nu t A} u_{0}+A^{1 / 2} \int_{0}^{t} e^{-\nu(t-\tau) A} A^{-1 / 2} P \operatorname{div} F(\tau) d \tau
$$

almost everywhere. We use $[14$, IV Theorems 2.3.1, 2.4.1] to obtain that $E$ lies in the Leray-Hopf class (1.2) and is a weak solution of the linear stationary Stokes system with data $f, u_{0}$. To finish the proof, we want to show that

$$
u \in L^{8}\left(0, T ; L^{4}(\Omega)\right) .
$$

The validity of the above property implies

$$
u \otimes u \in L^{2}\left(0, T ; L^{2}(\Omega)\right)
$$

As a consequence of (3.14) and (3.17) we conclude that $\tilde{u}$ lies in the LerayHopf class (1.2) and $\tilde{u}$ is the unique weak solution of the linear, stationary Stokes system with the external force $\operatorname{div}(u \otimes u)$ and vanishing initial value, see $[14$, IV Theorems 2.3.1, 2.4.1]. Furthermore, from these two Theorems and $\langle u \otimes u, \nabla u\rangle(\tau)=0$ almost everywhere, it follows that $u$ is, after a possible redefinition on a set of Lebesgue measure 0 , strongly continuous and satisfies the energy equality (1.14). 
Since in the case $q=4$ (and $s=8$ ) there is nothing left to be proved, we may assume in the proof of (3.16) that $q \neq 4$.

Assertion 1: $E=E_{1}+E_{2} \in L^{8}\left(0, T ; L^{4}(\Omega)\right)$.

Proof. In the case $4<q<\infty$ it is easily seen since $L_{\sigma}^{2}(\Omega) \cap L_{\sigma}^{q}(\Omega) \subset L_{\sigma}^{4}(\Omega)$ that $E_{1}(t)=e^{-\nu t A} u_{0}=e^{-\nu t A_{q}} u_{0} \in L^{8}\left(0, T ; L^{4}(\Omega)\right)$. If $3<q<4$ we use [11, Theorem 1.2 (ii)] to find a constant $c>0$, independent of $t$, such that

$$
\left\|e^{-\nu t A_{4}} u_{0}\right\|_{4} \leq c t^{-\frac{3}{2}\left(\frac{1}{q}-\frac{1}{4}\right)}\left\|u_{0}\right\|_{q}
$$

for all $t>0$. The estimate

$$
\int_{0}^{T}\left\|e^{-\nu t A_{4}} u_{0}\right\|_{4}^{8} d t \leq c\left\|u_{0}\right\|_{q}^{8} \int_{0}^{T} t^{-12\left(\frac{1}{q}-\frac{1}{4}\right)} d t<\infty
$$

implies $E_{1} \in L^{8}\left(0, T ; L^{4}(\Omega)\right)$. To get the property $E_{2} \in L^{8}\left(0, T ; L^{4}(\Omega)\right)$ we estimate for almost all $t \in[0, T[$, using $(2.7),(2.8)$ and (2.11), that

$$
\begin{aligned}
\left\|E_{2}(t)\right\|_{4} & \leq c\left\|A^{3 / 8} E_{2}(t)\right\|_{2} \\
& =c\left\|\int_{0}^{t} A^{7 / 8} e^{-\nu(t-\tau) A} A^{-1 / 2} P \operatorname{div} F(\tau) d \tau\right\|_{2} \\
& \leq c \nu^{-7 / 8} \int_{0}^{T}|t-\tau|^{-7 / 8}\|F(\tau)\|_{2} d \tau .
\end{aligned}
$$

Then an application of the Hardy-Littlewood inequality (2.18) yields

$$
\left\|E_{2}\right\|_{4,8, \Omega ; T} \leq c \nu^{-\frac{7}{8}}\|F\|_{2,4, \Omega ; T}<\infty
$$

Assertion 2: Let $3<q<4$. Then $\tilde{u} \in L^{8}\left(0, T ; L^{4}(\Omega)\right)$.

Proof. We use an iterative argument to improve the regularity in space step by step. Assume that for almost all $t \in\left[0, T\right.$ [ with certain parameters $s_{k}, r_{k}, \beta_{k}$

$$
\begin{aligned}
& \tilde{u}(t)=-\int_{0}^{t} A_{r_{k}}^{\beta_{k}} e^{-\nu(t-\tau) A_{r_{k}}} A_{r_{k}}^{-\beta_{k}} P_{r_{k}} \operatorname{div}((\tilde{u}+E) \otimes(\tilde{u}+E)) d \tau, \\
& \tilde{u}, E \in L^{s_{k}}\left(0, T ; L^{r_{k}}(\Omega)\right) \text { with } 3<r_{k}<4, \frac{2}{s_{k}}+\frac{3}{r_{k}}=1, \beta_{k} \in\left[\frac{1}{2}, 1\right] .
\end{aligned}
$$

For $k=1$ the iteration starts with $s_{1}:=s, r_{1}:=q$ and $\beta_{1}:=\frac{3}{2 q}+\frac{1}{2}=\alpha$, see (3.14). We denote by $r_{k+1}>r_{k}$ the unique element satisfying $\frac{1}{3}+\frac{1}{r_{k+1}}=$ $\frac{1}{r_{k} / 2}$ and set $s_{k+1}:=\frac{s_{k}}{2}$. Then (3.9) implies that

$$
\tilde{u} \in L^{s_{k+1}}\left(0, T ; L^{r_{k+1}}(\Omega)\right) .
$$

We define $\beta_{k+1}:=\frac{3}{r_{k+1}}-\frac{3}{2 r_{k+1}}+\frac{1}{2}=\frac{3}{2 r_{k+1}}+\frac{1}{2}$ and get with (3.7)

$$
\tilde{u}(t)=-\int_{0}^{t} A_{r_{k+1}}^{\beta_{k+1}} e^{-\nu(t-\tau) A_{r_{k+1}}} A_{r_{k+1}}^{-\beta_{k+1}} P_{r_{k+1}} \operatorname{div}((\tilde{u}+E) \otimes(\tilde{u}+E)) d \tau .
$$


From the first step of the proof we know that $E \in L^{8}\left(0, T ; L^{4}(\Omega)\right)$. There can occur two different possibilities. If $4 \leq r_{k+1}<\infty$ we get by an interpolation argument $\tilde{u}, E \in L^{8}\left(0, T ; L^{4}(\Omega)\right)$. Otherwise, if $3<r_{k+1}<4$, an interpolation argument yields $E \in L^{s_{k+1}}\left(0, T ; L^{r_{k+1}}(\Omega)\right)$. Looking at $(3.21),(3.22)$, we see that (3.19) and (3.20) are satisfied with the parameters $s_{k+1}, r_{k+1}, \beta_{k+1}$. Therefore, we can start a new step of this iterative argument. Repeating this step finitely many times, we get $\tilde{u} \in L^{8}\left(0, T ; L^{4}(\Omega)\right)$ which finishes the proof of Assertion 2.

Assertion 3: Let $4<q<\infty$. Then $\tilde{u} \in L^{8}\left(0, T ; L^{4}(\Omega)\right)$.

Proof. Assume that we have for almost all $t \in[0, T$ [ with certain parameters $s_{k}, r_{k}, \beta_{k}$

$$
\begin{aligned}
& \tilde{u}(t)=-\int_{0}^{t} A_{r_{k}}^{\beta_{k}} e^{-\nu(t-\tau) A_{r_{k}}} A_{r_{k}}^{-\beta_{k}} P_{r_{k}} \operatorname{div}((\tilde{u}+E) \otimes(\tilde{u}+E)) d \tau \\
& \tilde{u}, E \in L^{s_{k}}\left(0, T ; L^{r_{k}}(\Omega)\right) \quad \text { with } 4<r_{k}<\infty, \frac{2}{s_{k}}+\frac{3}{r_{k}}=1, \beta_{k} \in\left[\frac{1}{2}, 1\right] .
\end{aligned}
$$

Again, for $k=1$, the iteration starts with $s_{1}:=s, r_{1}:=q$ and $\beta_{1}:=\frac{3}{2 q}+\frac{1}{2}=\alpha$, see (3.14). We set $r_{k+1}:=\frac{3}{4} r_{k}$ and $\beta_{k+1}:=\frac{3}{r_{k}}-\frac{3}{2 r_{k+1}}+\frac{1}{2}=\frac{1}{r_{k}}+\frac{1}{2}$. Let $s_{k+1}>2$ be the unique element which satisfies the relation $\frac{2}{s_{k+1}}+\frac{3}{r_{k+1}}=1$. Then (3.7) implies that $\tilde{u}$ has the representation (3.22) with the new parameters $s_{k+1}, r_{k+1}, \beta_{k+1}$. From (3.22) we conclude with (3.8) that

$$
\tilde{u} \in L^{s_{k+1}}\left(0, T ; L^{r_{k+1}}(\Omega)\right) .
$$

From the first step of the proof we know that $E \in L^{8}\left(0, T ; L^{4}(\Omega)\right)$. There can occur two different possibilities. If $3<r_{k+1} \leq 4$ we get by an interpolation argument $\tilde{u}, E \in L^{8}\left(0, T ; L^{4}(\Omega)\right)$. Otherwise, if $4<r_{k+1}<\infty$, we use an interpolation argument to get $E \in L^{s_{k+1}}\left(0, T ; L^{r_{k+1}}(\Omega)\right)$. If we look at $(3.22)$, (3.25) we see that Eqs. (3.23) and (3.24) are satisfied with the parameters $s_{k+1}, r_{k+1}, \beta_{k+1}$. Therefore, we can start a new step of this iterative argument. Repeating this step finitely many times, we get $\tilde{u} \in L^{8}\left(0, T ; L^{4}(\Omega)\right)$ which finishes the proof of Assertion 3.

Now the claim (3.16) for $u=\tilde{u}+E$ follows, and the proof of this theorem is complete.

\section{Proof of regularity results}

Before proving Theorems 1.2 and 1.3 we need a useful, but technical lemma. In this lemma we assume that $u$ satisfies the strong energy inequality (1.5) to consider the term $u(t)$ for almost all $t \in[0, T]$ as initial value of a local strong 
solution which can be identified locally with $u$. Therefore, the proof will be based on Theorem 1.4. We will use the notation

$$
f_{a}^{b} f(x) d x:=\frac{1}{b-a} \int_{a}^{b} f(x) d x
$$

for the mean value of an integral.

Lemma 4.1. Let $\Omega, q, s, f, u_{0}, T$ satisfy the assumptions of Theorem 1.4, let $1 \leq s^{\prime} \leq s$, and let $u$ be a weak solution of (1.1) satisfying the strong energy inequality (1.5). Then there exists a constant $\epsilon_{*}=\epsilon_{*}\left(q, s^{\prime}, \Omega\right)>0$ with the following property: If $0<t_{0}<t \leq t_{1} \leq T$, and if

$$
\begin{gathered}
\int_{t_{0}}^{t_{1}}\|F(\tau)\|_{q *}^{s} d \tau \leq \epsilon_{*} \nu^{2 s-1}, \\
f_{t_{0}}^{t}\left(t_{1}-\tau\right)^{\frac{s^{\prime}}{s}}\|u(\tau)\|_{q}^{s^{\prime}} d \tau \leq \epsilon_{*} \nu^{s^{\prime}-\frac{s^{\prime}}{s}},
\end{gathered}
$$

then there exists a $\delta=\delta(t)>0$ such that $u \in L^{s}\left(t-\delta, t_{1} ; L^{q}(\Omega)\right)$. In particular, if $t_{1}>t$, then $t$ is a regular point of $u$.

Proof. We may assume that $u(\tau) \in L^{2}(\Omega)$ for all $\tau \in[0, T[$. From (4.2) and the fact that $u$ satisfies the strong energy inequality we find a null set $N \subset] t_{0}, t$ [ such that for $\left.\tau_{0} \in\right] t_{0}, t\left\lceil\backslash N\right.$ it holds $u\left(\tau_{0}\right) \in L_{\sigma}^{q}(\Omega)$ and

$$
\frac{1}{2}\left\|u\left(\tau_{1}\right)\right\|_{2}^{2}+\nu \int_{\tau_{0}}^{\tau_{1}}\|\nabla u\|_{2}^{2} d \tau \leq \frac{1}{2}\left\|u\left(\tau_{0}\right)\right\|_{2}^{2}-\int_{\tau_{0}}^{\tau_{1}}\langle F, \nabla u\rangle_{\Omega} d \tau
$$

for all $\tau_{1}$ mit $\tau_{0} \leq \tau_{1}<T$. Moreover, the condition (4.2) yields the existence of $\left.\tau_{0} \in\right] t_{0}, t[\backslash N$ which fulfills the inequality

$$
\left(t_{1}-\tau_{0}\right)^{\frac{s^{\prime}}{s}}\left\|u\left(\tau_{0}\right)\right\|_{q}^{s^{\prime}} \leq f_{t_{0}}^{t}\left(t_{1}-\tau\right)^{\frac{s^{\prime}}{s}}\|u(\tau)\|_{q}^{s^{\prime}} d \tau \leq \epsilon_{*} \nu^{s^{\prime}-\frac{s^{\prime}}{s}} .
$$

It follows with a constant $c=c(\Omega, q)>0$ that

$$
\begin{aligned}
\int_{0}^{t_{1}-\tau_{0}}\left\|e^{-\nu \tau A_{q}} u\left(\tau_{0}\right)\right\|_{q}^{s} d \tau & \leq \int_{0}^{t_{1}-\tau_{0}} c\left\|u\left(\tau_{0}\right)\right\|_{q}^{s} d \tau \\
& =c\left(t_{1}-\tau_{0}\right)\left\|u\left(\tau_{0}\right)\right\|_{q}^{s} \leq c \epsilon_{*}^{\frac{s}{s^{\prime}}} \nu^{s-1} .
\end{aligned}
$$

Hence with a new constant $\widetilde{\epsilon_{*}}:=\left(\frac{\epsilon_{*}}{c}\right)^{\frac{s^{\prime}}{s}}$, where $\epsilon_{*}$ is the constant from Theorem 1.4, the conditions of Theorem 1.4 are satisfied. We get the existence of a unique weak solution $v \in L^{s}\left(\left[\tau_{0}, t_{1}\left[; L_{\sigma}^{q}(\Omega)\right)\right.\right.$ to the Navier-Stokes system (1.1) with initial value $v\left(\tau_{0}\right)=u\left(\tau_{0}\right)$. Considering $u$ as a weak solution to the Navier-Stokes system with initial value $u\left(\tau_{0}\right)$ on $\left[0, t_{1}-\tau_{0}\right]$, we use Serrin's Uniqueness Theorem to get that $u=v \in L^{s}\left(\tau_{0}, t_{1} ; L_{\sigma}^{q}(\Omega)\right)$. The proof is complete. 
Proof of Theorem 1.2. (1) Because of (1.6) it is possible to choose a $\delta>0$ such that with $t_{0}:=t-\delta, t_{1}:=t+\delta$ the estimate

$$
\begin{aligned}
f_{t-\delta}^{t}\left(t_{1}-\tau\right)^{\frac{s^{\prime}}{s}}\|u(\tau)\|_{q}^{s^{\prime}} d \tau & \leq \frac{1}{\delta} \int_{t-\delta}^{t}(2 \delta)^{\frac{s^{\prime}}{s}}\|u(\tau)\|_{q}^{s^{\prime}} d \tau \\
& =\frac{2^{\frac{s^{\prime}}{s}}}{\delta^{1-\frac{s^{\prime}}{s}}} \int_{t-\delta}^{t}\|u(\tau)\|_{q}^{s^{\prime}} d \tau \leq \epsilon_{*} \nu^{s^{\prime}-\frac{s^{\prime}}{s}}
\end{aligned}
$$

holds. This shows (4.2). Furthermore, condition (4.1) on $F$ can be fulfilled as well. Then Lemma 4.1 proves the sufficiency of (1.6) to imply regularity of $u$ at $t$.

(2) The constant $\epsilon_{*}=\epsilon_{*}(q, \Omega)>0$ will be determined in the proof; therefore, we begin with considering $\epsilon_{*}$ as an arbitrary, fixed positive number. Let $\varepsilon_{1}=\varepsilon_{1}(q, \Omega)>0$ denote the constant from Theorem 1.4 which in (1.12), (1.13) is called $\epsilon_{*}$, and let $\epsilon_{2}=\epsilon_{2}\left(s^{\prime}, \Omega\right)$ be the constant in Lemma 1.5 called $\epsilon_{*}$ in (4.1), (4.2). We assume $\epsilon_{*} \leq \varepsilon_{1}$ and $u_{0} \neq 0$. It holds

$$
\int_{0}^{\delta_{1}}\left\|e^{-\nu \tau A_{q}} u_{0}\right\|_{q}^{s} d \tau \leq c \delta_{1}\left\|u_{0}\right\|_{q}^{s}, \quad c=c(\Omega, q)>0 .
$$

We define

$$
\delta_{1}:=\min \left(\frac{\varepsilon_{1} \nu^{s-1}}{c\left\|u_{0}\right\|_{q}^{s}}, T\right) .
$$

If $\delta_{1}=T$, we already know that $u \in L^{s}\left(0, T ; L^{q}(\Omega)\right)$. So let us assume that $\delta_{1}=\frac{\varepsilon_{1} \nu^{s-1}}{c\left\|u_{0}\right\|_{q}^{s}}$. With this choice of $\delta_{1}$, Theorem 1.4 yields the existence of a unique weak solution $v \in L^{s}\left(0, \delta_{1} ; L^{q}(\Omega)\right)$ of (1.1), which coincides by Serrin's Uniqueness Theorem with $u$ on $\left[0, \delta_{1}\left[\right.\right.$. For an arbitrary $t \in\left[\frac{\delta_{1}}{2}, T-\frac{\delta_{1}}{2}\right]$, we get with $t_{0}:=t-\frac{\delta_{1}}{2}, t_{1}:=t+\frac{\delta_{1}}{2}$

$$
\begin{aligned}
& f_{t_{0}}^{t}\left(t_{1}-\tau\right)^{\frac{s^{\prime}}{s}}\|u(\tau)\|_{q}^{s^{\prime}} d \tau \leq \frac{2}{\delta_{1}^{1-\frac{s^{\prime}}{s}}} \int_{0}^{T}\|u(\tau)\|_{q}^{s^{\prime}} d \tau \\
& \quad \leq 2\left(\frac{\varepsilon_{1} \nu^{s-1}}{c\left\|u_{0}\right\|_{q}^{s}}\right)^{\frac{s^{\prime}}{s}-1} \epsilon_{*} \frac{\nu^{s-1}}{\left\|u_{0}\right\|_{q}^{s-s^{\prime}}}=2\left(\frac{\varepsilon_{1}}{c}\right)^{\frac{s^{\prime}}{s}-1} \epsilon_{*} \nu^{s^{\prime}-\frac{s^{\prime}}{s}} .
\end{aligned}
$$

From this estimate it follows that we may define

$$
\epsilon_{*}:=\min \left(\frac{\varepsilon_{2}}{2}\left(\frac{\varepsilon_{1}}{c}\right)^{1-\frac{s^{\prime}}{s}}, \varepsilon_{1}, \varepsilon_{2}\right) .
$$

We see that $\epsilon_{*}$ depends only on $\Omega, q, s^{\prime}$. Using Lemma 4.1 we find $\delta=\delta(t)>0$ such that

$$
u \in L^{s}\left(t-\delta(t), t+\frac{\delta_{1}}{2} ; L^{q}(\Omega)\right) .
$$

With (4.7) and $u \in L^{s}\left(0, \delta_{1} ; L^{q}(\Omega)\right)$ we obtain due to the compactness of the interval $[0, T]$ that $u \in L^{s}\left(0, T ; L^{q}(\Omega)\right)$.

Now the theorem is completely proved. 
Proof of Theorem 1.3. By interpolation, in both cases the weak solution $u$ satisfies $u \in L^{s^{\prime}}\left(0, T ; L^{q}(\Omega)\right)$. The idea of the proof is to use Lemma 4.1. To control the term in (4.2) we use the interpolation inequality, see [1, Theorem 4.3.1],

$$
\|v\|_{q} \leq c\|v\|_{2}^{1-\frac{2}{s^{\prime}}}\|\nabla v\|_{2}^{\frac{2}{s^{\prime}}}, \quad v \in H_{0}^{1}(\Omega)
$$

where $c=c(\Omega, q)>0$. For $\delta \in] 0, \delta_{0}\left[\right.$ with a small $\delta_{0}>0$ we get with $t_{0}:=t-\delta$, $t_{1}:=t+\delta$ the estimate

$$
\begin{aligned}
I(\delta) & :=f_{t-\delta}^{t}\left(t_{1}-\tau\right)^{\frac{s^{\prime}}{s}}\|u(\tau)\|_{q}^{s^{\prime}} d \tau \\
& \leq c \delta^{\frac{s^{\prime}}{s}-1} \int_{t-\delta}^{t}\left(\|u(\tau)\|_{2}^{1-\frac{2}{s^{\prime}}}\|\nabla u(\tau)\|_{2}^{\frac{2}{s^{\prime}}}\right)^{s^{\prime}} d \tau \\
& \leq c \delta^{\frac{s^{\prime}}{s}-1}\|u\|_{2, \infty ; T}^{s^{\prime}-2} \int_{t-\delta}^{t}\|\nabla u(\tau)\|_{2}^{2} d \tau
\end{aligned}
$$

with a constant $c=c(\Omega, q)>0$. Since $u$ is supposed to satisfy the strong energy inequality (1.5), we may proceed for almost all $\delta \in] 0, \delta_{0}[$ as follows:

$$
I(\delta) \leq \frac{c}{\nu} \delta^{\frac{s^{\prime}}{s}-1}\left(|E(t-\delta)-E(t)|+\left|\int_{t-\delta}^{t}\langle f, u\rangle d \tau\right|\right)
$$

where the constant $c$ depends on $\|u\|_{2, \infty ; T}$ in the case $\alpha>\frac{1}{2}$ and $c=c(\Omega)$ if $\alpha=\frac{1}{2}$. By Hölder's inequality we get that

$$
\left|\frac{1}{\delta^{\frac{s^{\prime}}{4}}} \int_{t-\delta}^{t}\langle f(\tau), u(\tau)\rangle d \tau\right| \leq\|u\|_{2, \infty ; T}\left(\int_{t-\delta}^{t}\|f\|_{2}^{\frac{4}{4-s^{\prime}}} d \tau\right)^{\frac{4-s^{\prime}}{4}} .
$$

As $\frac{s}{s^{\prime}}=\frac{4}{4-s^{\prime}}$ and consequently $f \in L^{\frac{4}{4-s^{\prime}}}\left(0, T ; L^{2}(\Omega)\right)$, the left-hand side in the previous inequality converges to 0 as $\delta \rightarrow 0+$.

First consider the case $\alpha>\frac{1}{2}$ and choose $\epsilon>0$ with $s^{\prime}=4 \alpha-\epsilon$. Due to the assumption (1.8) we get with $1-\frac{s^{\prime}}{s}=\frac{s^{\prime}}{4}=\alpha-\frac{\epsilon}{4}$

$$
\lim _{\delta \rightarrow 0+} \frac{c}{\nu} \delta^{-\frac{s^{\prime}}{4}}|E(t-\delta)-E(t)|=\lim _{\delta \rightarrow 0+} \frac{c}{\nu} \delta^{\frac{\epsilon}{4}} \frac{|E(t-\delta)-E(t)|}{\delta^{\alpha}}=0 .
$$

Consequently the right hand side of (4.9) converges to 0 as $\delta \rightarrow 0+$. Hence we can fulfill (4.2) and, due to the assumption $F \in L^{s}\left(0, T ; L^{r}(\Omega)\right)$, it is also possible to satisfy (4.1). Altogether, Lemma 4.1 yields regularity of $u$ at $t$.

Secondly, consider the case $\alpha=\frac{1}{2}$ in which $s^{\prime}=2, s=4$. We will choose the constant $\gamma_{*}=\gamma_{*}(\Omega)>0$ below. Let $\epsilon_{*}=\epsilon_{*}(q)>0$ denote the constant from Lemma 4.1. The assumption (1.9) implies that for all $0<\delta<\mu$

$$
\frac{1}{\nu} \frac{|E(t-\delta)-E(t)|}{\delta^{\frac{1}{2}}} \leq \gamma_{*} \nu^{\frac{3}{2}}
$$


Then by (4.9), (4.10) and (4.12) we get with a constant $c=c(\Omega)>0$ for almost all $\delta \in] 0, \delta_{0}[$ that

$$
I(\delta) \leq c \gamma_{*} \nu^{\frac{3}{2}}+\frac{c}{\nu}\|u\|_{2, \infty ; T}\left(\int_{t-\delta}^{t}\|f\|_{2}^{2} d \tau\right)^{\frac{1}{2}} .
$$

Now with $\gamma_{*}:=\frac{\epsilon_{*}}{2 c}$ we find $0<\delta<\mu$ such that $I(\delta) \leq \epsilon_{*} \nu^{\frac{3}{2}}$, cf. (4.2), and that (4.1) is satisfied. Hence Lemma 4.1 implies regularity of $u$ at $t$.

\section{References}

[1] Adams, R., Fournier, J.: Sobolev Spaces. Academic Press, New York (2003)

[2] Farwig, R., Kozono, H., Sohr, H.: Local in time regularity properties of the Navier-Stokes equations. Indiana Univ. Math. J. 56, 2111-2131 (2007)

[3] Farwig, R., Kozono, H., Sohr, H.: Very weak solutions of the Navier-Stokes equations in exterior domains with nonhomogeneous data. J. Math. Soc. Japan 59, 127-150 (2007)

[4] Farwig, R., Kozono, H., Sohr, H.: Very weak, weak and strong solutions to the Navier-Stokes system. Topics on partial differential equations, In: Kaplický, P., Nečasová, Š. (eds.) J. Nečas Center Math. Modeling, Prague. Lecture Notes, vol. 2, pp. 1-54 (2007)

[5] Farwig, R., Kozono, H., Sohr, H.: Energy-based regularity criteria for the Navier-Stokes equations. J. Math. Fluid Mech. 11, 1-14 (2008)

[6] Farwig, R., Kozono, H., Sohr, H.: Criteria of local in time regularity of the Navier-Stokes equations beyond Serrin's condition. Banach Center Publ. 81, 175-184 (2008)

[7] Farwig, R., Sohr, H.: Generalized resolvent estimates for the Stokes system in bounded and unbounded domains. J. Math. Soc. Japan 46, 607-643 (1994)

[8] Galdi, G.P. : An introduction to the Navier-Stokes initial value problem. In: Galdi, G.P. (ed.) Fundamental Directions in Mathematical Fluid Mechanics, pp. 1-70. Birkhäuser, Basel (2000)

[9] Giga, Y., Sohr, H.: On the Stokes operator in exterior domains. J. Fac. Sci. Univ. Tokyo, Sec. IA 36, 103-130 (1989)

[10] Giga, Y., Sohr, H.: Abstract $L^{p}$ estimates for the Cauchy problem with applications to the Navier-Stokes equations in exterior domains. J. Funct. Anal. 102, 72-94 (1991)

[11] Iwashita, H.: $L_{q}-L_{r}$ estimates for solutions of the nonstationary Stokes equations in an exterior domain and the Navier-Stokes initial value problems in $L_{q}$ spaces. Math. Ann. 285, 265-288 (1989) 
[12] Komo, C.: Regularität von schwachen Lösungen der Navier-Stokes-Gleichungen im Außengebiet, Diploma Thesis, Darmstadt University of Technology, Darmstadt (2009)

[13] Miyakawa, T., Sohr, H.: On energy inequality, smoothness and large time behavior in $L^{2}$ for weak solutions of the Navier-Stokes equations in exterior domains. Math. Z. 199, 455-478 (1988)

[14] Sohr, H.: The Navier-Stokes-Equations: An Elementary Functional Analytic Approach. Birkhäuser, Basel (2001)

R. Farwig, C. Komo

Fachbereich Mathematik,

Technische Universität Darmstadt, 64289 Darmstadt,

Germany

e-mail: farwig@mathematik.tu-darmstadt.de

C. Komo

e-mail: komo@mathematik.tu-darmstadt.de

Received: 15 June 2009.

Accepted: 07 January 2010. 